\title{
Schlagwort «Grundversorger-Mangel»
}

Lukas R. Meier

Dr. med., Co-Präsident der Gesellschaft der Ärztinnen und Ärzte des Kantons Solothurn (GAeSO)
Korrespondenz:

Dr. med. Lukas R. Meier

Kantonsspital

CH-4600 Olten

Tel. 0623114343

Fax 0623114337

Imeier_ol@spital.ktso.ch
In allen Medien wird repetiert, von diversen politischen Ecken gemahnt, von Journalisten beschworen: «Das Ende der Grundversorger, das grosse Hausarztsterben naht. Wo bleibt der Nachwuchs, kommt es zu medizinischen Versorgungslücken?»

Wer versorgt unsere chronischkranken Mitmenschen? Wer kümmert sich um die Bagatell-Unfälle, den Schnupfen und wer impft gegen die Grippe? Wer führt die Patienten durch den Dschungel der Spezialisten?

Nach Lösungen wird gesucht: Junge Mediziner werden bereits im Studium motiviert, es werden Praktika beim Hausarzt angeboten. Man versucht, die Taxpunktwerte in abgelegenen Tälern und auf dem Land zu erhöhen, man verspricht sich Hilfe durch den Aufbau von Gesundheitszentren, wo durch die Feminisierung der Medizin in Teilarbeitszeitmodellen gearbeitet werden kann. Die Spitäler rüsten ihre Notfallstationen mit integrierten Hausarztpraxen auf. Die Gesundheitspolitiker schwören auf Managed Care, und da dieser Begriff bereits abgenutzt ist, auf die Integrierte Versorgung.

Mit dem Modell Integrierte Versorgung bzw. Managed Care möchten findige Immobilienmakler in ihren teuren Neubauten Gesundheitszentren realisieren. Dass sie keine Ärzte der Grundversorgung finden, merken sie erst nach mehreren Inserat-Leerläufen.

Und ganz still, auf leisen Sohlen, erscheinen im Gesundheitsmarkt andere Strukturen. Es scheint sich abzuzeichnen, dass wir vom ärztezentrierten Gesundheitssystem abweichen (müssen). Wir werden den Begriff des «lower use of health care services» bald häufiger zu lesen bekommen.

Ein paar Beispiele gefällig?

\section{Aufwertung der MPA}

Das nichtärztliche Personal, spezifisch weitergebildet, soll eine Basisfunktion in der Betreuung der Chronischkranken-Patientengruppe übernehmen.

Das Seminar «Betreuung von LangzeitpatientenModul Diabetes» wird vom MPA-Verband bereits seit 2001 angeboten. Seit 2009 existiert ein entsprechendes Modul für Rheuma. In der CARAT-Studie sollen die Resultate unter Einbezug der MPA in der Einstellung des HbA1c, LDL und Blutdruck geprüft werden.

Diese Weiterbildung der MPA soll schliesslich ab 2012 in einen eidgenössischen Fachausweis in Chronic Care Management münden, mit den Befähigungen, z. B. COPD, Rheuma, Herzinsuffizienz, Koronare Herzkrankheit, Diabetes, Adipositas, Bluthochdruck zu managen, immer natürlich mit der Unterstützung des Allgemeinpraktikers im Hintergrund bzw. im Team.

Knackpunkt dieser an sich vernünftigen Entwicklung ist die finanzielle Abgeltung mit speziellen (nicht-ärztlichen) Taxpunktwerten. Bisher ist die MPA in der Arztpraxis unter «Inventar» und «Praxisunkosten» subsumiert.

Die Aufwertung der MPA, sei es im Chronic-CareManagement oder als Diabetes-Betreuung usw. scheint mir die einzig wegweisende Lösung zu sein. Die räumliche Nähe zum Grundversorger, um bei Problemen sofort reagieren zu können, ist ideal. Voraussetzung wäre, wie oben erwähnt, die korrekte finanzielle Abgeltung.

\section{Wundexpertin, Pflegeexpertin Stoma- Inkontinenz-Wunde}

Diese zwei Berufszweige, die in den meisten Fällen von diplomierten Pflegefachfrauen, zum Teil mit höherer Fachausbildung, ausgeübt werden, sind bereits seit Jahren etabliert.

Die diplomierten Wundexpertinnen SAFW (Lehrgang: ca. 24 Tage) sind mit den neusten Erkenntnissen der Wundbehandlung einschliesslich eines effizienten Wundmanagements vertraut. Sie gewährleisten Patientinnen und Patienten eine kontinuierliche, professionelle Wundbehandlung und Wundberatung bei chronischen Wunden.

Die Stomatherapeutinnen spezialisieren sich durch eine angemessene Ausbildung und durch eine jahrelange praktische Erfahrung. Neuerdings tragen sie die Berufsbezeichnung Pflegeexperte Stoma-Inkontinenz-Wunde. Diese Ausbildung wird in Deutschland angeboten (DVET Fachverband Stoma und Inkontinenz e.V.; www.stoma-ch.com/index.php?page=192).

Die Abgeltung ist in diesen zwei Fachgebieten durch den TARMED geregelt. Meistens werden diese Leistungen in den Spitälern angeboten, zunehmend aber auch freiberuflich.

Die diplomierten Wund- und Stomaexpertinnen würde man ebenso gerne an einem Gesundheitszentrum angesiedelt sehen, da auch hier, wie die Erfahrung am Beispiel Spital-Wundambulatorium zeigt, die schnelle Zuziehung des (Fach-)Arztes in Zweifelsfällen günstig sein kann.

\section{Telemedizin und Telefonberatung in der Apotheke, Telebiometrie/Telelabor} Im Moment auf grössere Skepsis stösst ein bisher nicht veröffentlichtes Pilot-Projekt: Der Patient holt 
sich die ärztliche Hilfe beim Apotheker, der mit dem Telefondoktor der Firma Medgate zusammenarbeitet. Die Idee ist grundsätzlich bestechend: Dem Apotheker fehlt die vollständige medizinische Ausbildung und der Telefondoktor kann nicht auf die eiternde Wunde oder in den Hals schauen. Zusammen werden die beiden aber zum schlagkräftigen Team. Viele Fragen sind jedoch noch offen: Kompetenzgrenzen, Sprechzimmer in der Apotheke zur Wahrung der Diskretion usw.

Die Telemedizin hingegen ist schon mehrere Jahre etabliert. Gemäss Medgate werden pro Tag bis zu 4300 Telefonkonsultationen abgewickelt. Bei entsprechenden Versicherungsmodellen sind diese Konsultationen für den Patienten gratis.

Die Telebiometrie geht einen Schritt weiter im Management der Chronischkranken. Hier erfolgt die kontinuierliche Messung, Überwachung und Auswertung der eingegangenen Messwerte und ist Grundlage der kontinuierlichen Therapieanpassung. Das Alarmmanagement gewährleistet ein zeitnahes Reagieren auf Entgleisungen der Messparameter bei Überschreitungen von standardisierten und individualisierten Grenzwerten.

Die Telemedizin hat die Bewährungsprobe bestanden. Die computergestützten Diagnose- bzw. Algorhythmen werden in Zukunft immer genauer und hilfreicher. Leider werden sie aber psychische bzw. psychosomatische Beschwerden kaum erkennen können.

Die «Zwischenschaltung» des Apothekers finde ich persönlich eine unglückliche Lösung, da sie die Beziehung Arzt-Patient eher verkompliziert und kaum Nutzen bringt.

\section{Diplomierter Psychologischer Patienten-Coach IKP}

Gemäss Ausbildungsplan soll dieses Berufsbild in eine Lücke zwischen Grundversorger/Spital-Krankenpflege und Psychologie springen, bei denen mehr und mehr zeitliche Ressourcen fehlen:

Der diplomierte Patienten-Coach IKP unterstützt Menschen, die mit einer schwerwiegenden Diagnose oder chronischen Krankheit konfrontiert sind. Man versucht dabei, die eigenen Kräfte und Fähigkeiten wieder zu mobilisieren. Durch Ermutigung und Empowerment wird dem Patienten ermöglicht, aus seiner Resignation und Passivität herauszufinden. Diese Zusammenarbeit zwischen Coach und Patient ist eine Hilfe zur Selbsthilfe und vermittelt dem Patienten ein Selbstverständnis, das ihm eine neue Form von Freiheit und Selbstbestimmung ermöglicht. Die Finanzierung des Patienten-Coach ist (noch) unklar.

Mir persönlich ist noch nicht klar, in welche Richtung sich der zukünftige Patienten-Coach bewegen soll. Er befindet sich (noch) irgendwo im Vakuum zwischen Arzt (oder Psychologe), Pflegefachfrau, Sozialdienst und Spitex sowie den diversen Selbsthilfegrup- pen. Ich bin aber überzeugt, dass sich ein Coach erübrigen könnte, wenn die zeitlichen Ressourcen der Grundversorger besser werden.

\section{EURO-doc}

Im Rahmen der Nivellierung Hochschulabschlüsse werden zunehmend zweistufige Systeme auftreten, bezeichnet als Bakkalaureus/Bachelor und Magister/ Master. Es ist abzusehen, dass auch ein Bachelor of Medicine (BM) als Studienabgänger anerkannt wird, der abschätzig bereits jetzt den Übernamen EUROdoc oder Barfussarzt hat. Auf grosse Kritik stossen solche Pläne noch innerhalb der Ärzteschaft, da weder ein Bedarf noch ein Berufsbild existiert.

Bachelor of Medicine und Bachelor of Surgery sind ausserhalb Europas, insbesondere in den USA, Kanada und Australien, bekannt. Es stellt sich die Frage, wo das Berufsbild eines Bachelor of Medicine Platz finden soll: als Spitalarzt, als wissenschaftlich tätiger Pflegefachmann, als Spezialist in ausgewählten Fachgebieten (in Konkurrenz zu den MPA), oder werden es andere Aufgaben sein?

\section{Diverse Körpertherapeuten}

Aufschlussreich zu diesem Thema ist ein Blick in das Register der Erfahrungs-Medizin, www.emindex.ch/ _d/methoden.las - Bachblütentherapie, AyurvedaMassage, Shin Tai, Ortho-Bionomy, Ohrkerzentherapie sind nur einige von ca. 200 Therapieformen, die das Register der Erfahrungsmedizin aufzählt. Wer von der Zusatzversicherung anerkannt werden will, und das wird vorläufig nur die Komplementärmedizin sein, muss ein Qualitätslabel anstreben, das klare Voraussetzungen bei Ausbildung, Fortbildung und Erfahrung fordert. Jeder Versicherer ist frei, diese Therapien zu vergüten. Erstaunlich ist die Tatsache, dass Patientinnen und Patienten offenbar bereit sind, solche Zusatzversicherungen abzuschliessen bzw. diese Angebote privat zu bezahlen, und gleichzeitig fordern, dass noch mehr dieser Methoden der obligatorischen Krankenversicherung unterstellt werden. Umso erstaunlicher, dass die gleichen Leute laut stöhnen, wenn die Prämien der obligatorischen Krankenversicherung steigen. Irritierend ist jedenfalls die $\mathrm{Zu}-$ nahme dieser erfahrungsmedizinischen Therapieformen, die bald an jeder Hausecke anzutreffen sind.

Die Körpertherapeuten und -therapeutinnen mit ihren vielfältigen Angeboten werden ihren Platz behaupten können. Sie werden aber ständigem Wandel ausgesetzt sein, da ihr Zielpublikum immer wieder neue, schlagwortgewichtige Therapieformen sucht und sich mit Altgedientem nicht mehr zufrieden gibt.

Die Zukunft wird zeigen, ob diese Wege unser Gesundheitssystem revolutionieren oder ruinieren. Jedenfalls sucht sich die Gesellschaft ihre eigenen Wege zum Wohlergehen. 